\title{
Anomalies de la muqueuse buccale révélant un purpura thrombopénique immunologique
}

\author{
Charles Albisetti ${ }^{1}$, Marc Baranes2, Louis Maman ${ }^{3}$ \\ Interne chirurgie orale, Département oral surgery, Paris 6, France \\ Praticien attaché, hôpital Charles Foix, Ivry-sur-Seine et pratique privée en chirurgie orale, Saint-Mandé, France \\ Professeur d'université, praticien hospitalier, doyen de la faculté d'odontologie Paris-Descartes, Paris, France \\ Correspondance : charlesalbisetti1@gmail.com
}

(Reçu 4 juin 2014, accepté le 18 août 2015)

Mots clés : purpura / thrombopénie / auto-immun

Key words: purpura / thrombopenia / autoimmune
Résumé - Introduction : Les lésions hémorragiques de la muqueuse buccale sont les manifestations cliniques les plus communes du purpura thombopénique immunologique (PTI). Ces manifestations amènent parfois le patient à consulter son chirurgien-dentiste en première intention. Observation : Une patiente de 37 ans a consulté aux urgences dentaires pour des gingivorragies spontanées et des lésions muqueuses intrabuccales. L'examen clinique révélait un syndrome hémorragique cutanéo-muqueux généralisé avec un hématome de la face interne de la joue droite. Une NFS réalisée en urgence montrait une thrombopénie sévère à $4 \mathrm{G} / \mathrm{L}$ associée à une anémie normocytaire. L'enquête étiologique excluait une cause centrale et ne retrouvait aucune cause périphérique. Le diagnostic de PTI a été posé. Un traitement à base de prednisone $1 \mathrm{mg} / \mathrm{kg}$ par jour et une perfusion d'immunoglobulines IV (Tégéline ${ }^{\circledR}$ ) ont permis une régression totale des symptômes et une normalisation du taux de plaquettes. Discussion : La connaissance des manifestations orales du PTI doit permettre au chirurgien-dentiste d'évoquer le diagnostic afin d'orienter le patient vers une structure médicale adaptée à la prise en charge en urgence.

\begin{abstract}
Disorders of the buccal mucosa revealing immune thrombocytopenic purpura. Introduction: Spontaneous gingivorrhagia is the most common oral manifestation of immune thrombocytopenic purpura. For this reason, the dentist is sometimes consulted first. Observation: A female patient, 37 years old, consulted at the dental emergency unit for spontaneous gingival bleeding and intraoral mucosal lesions. Clinical examination revealed mucocutaneous hemorrhagic syndrome with widespread bruising on the inner side of the right cheek. The blood count carried out in the emergency unit showed severe thrombocytopenia at $4 \mathrm{G} / \mathrm{L}$ associated with normocytic anemia. The etiological survey excluded a central cause of the thrombocytopenia and could not find any peripheric origin. The final diagnosis was immune thrombocytopenic purpura. A treatment based on prednisolone $1 \mathrm{mg} / \mathrm{kg}$ daily and IV immunoglobulin infusion (Tégéline ${ }^{\circledR}$ ) allowed complete resolution of the symptoms and normalization of the platelet count. Discussion: For the dentist, knowing the oral manifestations of immune thrombocytopenic purpura can be useful to guide the patient toward an emergency medical center.
\end{abstract}

\section{Introduction}

Le purpura thrombopénique immunologique (PTI) est une affection auto-immune rare et de cause inconnue aux cours de laquelle les plaquettes sont la cible erronée du système immunitaire. La sévérité de la thrombopénie peut parfois engager le pronostic vital par hémorragie cérébro-méningée, digestive ou génitale [1]. Les manifestations buccales du PTI sont essentiellement des gingivorragies spontanées. Elles peuvent amener le patient à consulter son chirurgien-dentiste. La connaissance de cette entité pathologique doit permettre au praticien d'évoquer le diagnostic afin d'orienter au plus vite le patient vers une structure médicale adaptée.

Nous rapportons le cas d'une patiente ayant consulté aux urgences odontologiques devant l'apparition d'un tableau hémorragique cutanéo-muqueux à prédominance buccale.

\section{Observation}

Une patiente de 37 ans s'est présentée au service d'odontologie pour des gingivorragies spontanées ainsi que des lésions 


\section{CAS C L I N I Q U E}

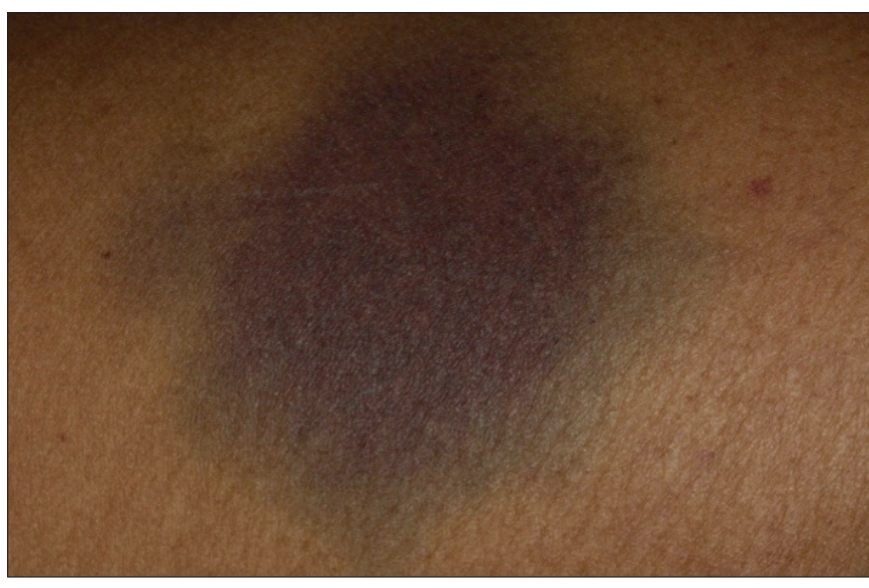

Fig. 1. Ecchymose de la jambe droite.

Fig. 1. Ecchymosis on the right leg.

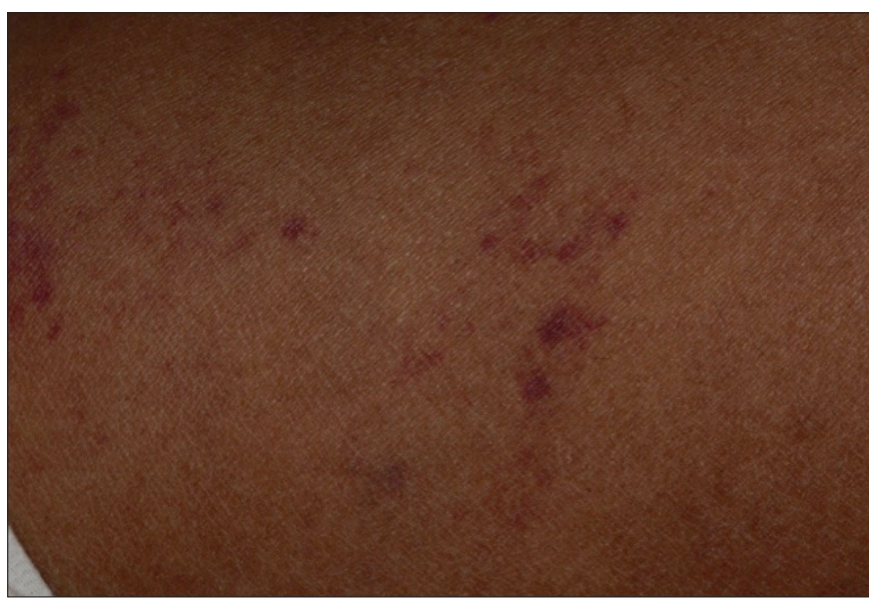

Fig. 2. Pétéchies de l'épaule gauche.

Fig. 2. Petechia on the left shoulder.

muqueuses érythémateuses intrabuccales. Elle avait pour antécédent une césarienne, une fausse couche spontanée et prenait une contraception orale par Microval ${ }^{\circledR}$ (lévonorgestrel), un comprimé par jour. Elle nous a confié avoir arrêté sa pilule la veille. La patiente était d'origine algérienne, aucune intoxication alcoolo-tabagique n'a été relevée. Les gingivorragies étaient apparues depuis 48 heures, une épistaxis était survenue la veille de la consultation.

L'examen général a révélé une ecchymose au niveau de la jambe droite (Fig. 1) survenue suite à un traumatisme mineur au travail ainsi qu'un purpura pétéchial à l'épaule gauche (Fig. 2) caractérisé par une trentaine de lésions punctiformes rouges et violacées ne s'effaçant pas à la vitropression.

L'examen exobuccal (Fig. 3) a mis en évidence quatre pétéchies isolées de l'angle labial gauche et trois petites ulcérations douloureuses de la lèvre inférieure du côté droit.

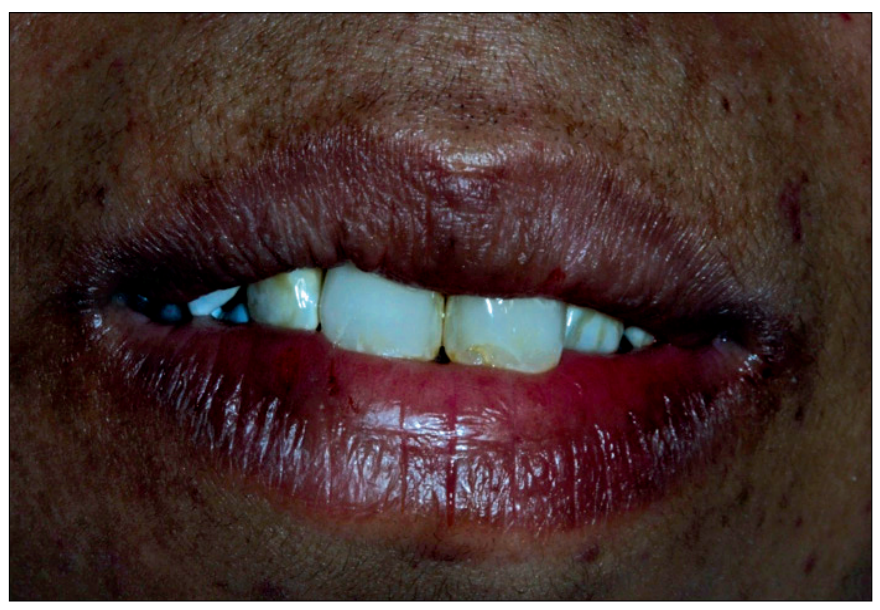

Fig. 3. Vue exobuccale. Fig. 3. Exobuccal view.

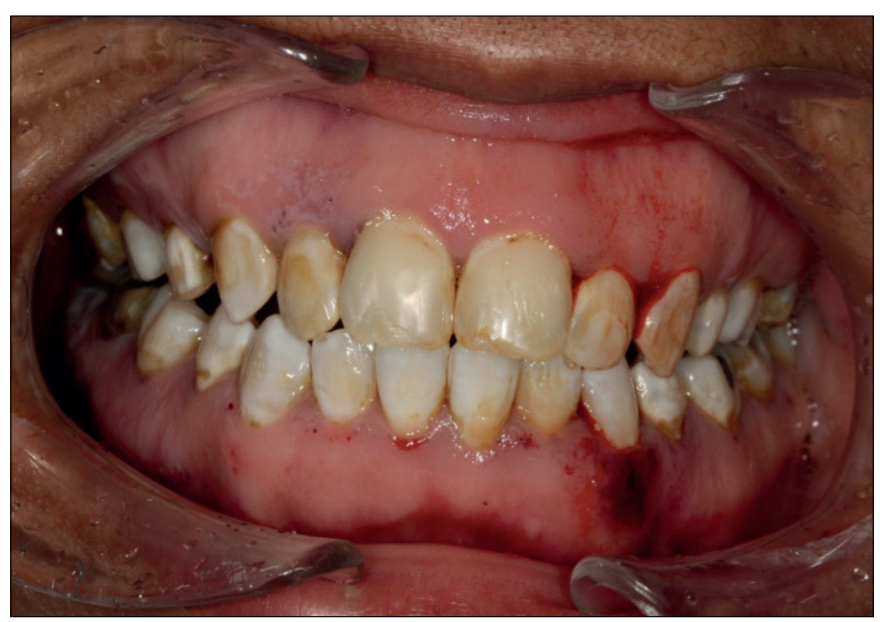

Fig. 4. Vue endobuccale.

Fig. 4. Endobuccal view.

L'examen endobuccal (Fig. 4) a retrouvé des gingivorragies spontanées dans tous les secteurs, une ecchymose sous le collet de la 33 et de nombreuses pétéchies sous le secteur incisivo-canin mandibulaire. À la face interne de la lèvre inférieure (Fig. 5), deux lésions ecchymotiques supérieures à $5 \mathrm{~mm}$ de diamètre de couleur rouge vif et 15 pétéchies de diamètre variable sur une muqueuse saine ont été observées. La palpation était indolore, les lésions ne s'effaçaient pas à la vitropression. Au niveau de la face interne de la joue droite (Fig. 6), on pouvait observer un hématome d'environ 2,5 cm de diamètre à mi-hauteur associé à un tic de mordillement, une vésicule en regard de la face occlusale de la 18 de 5 millimètres entourée d'un liseré érythémateux et une plage de lésions purpuriques. Au palais (Fig. 7), il existait de très nombreuses pétéchies s'étendant jusqu'au voile. 


\section{A S C L I N I Q U E}

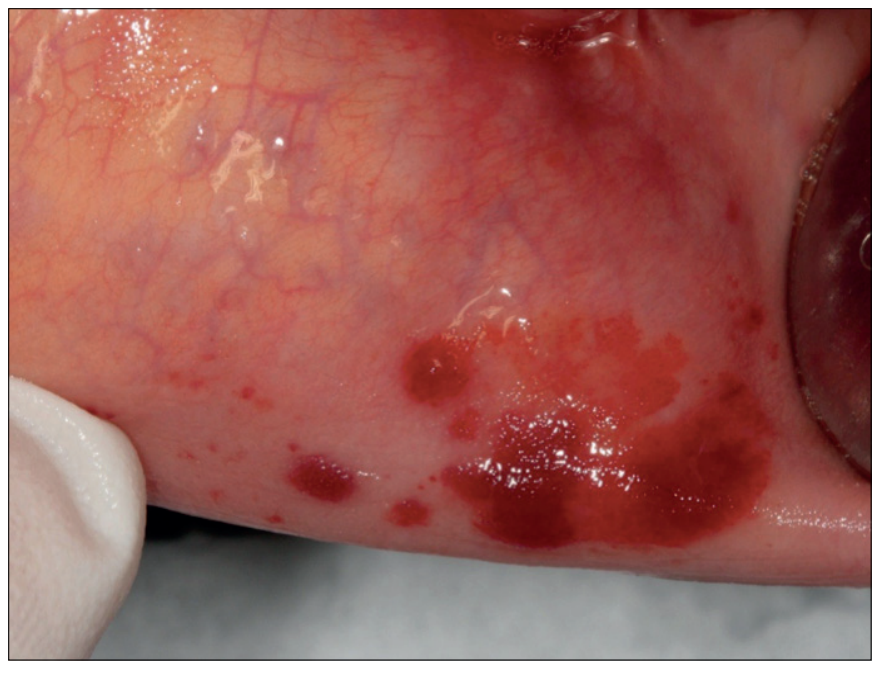

Fig. 5. Face interne de la lèvre inférieure.

Fig. 5. Internal side of inferior lip.

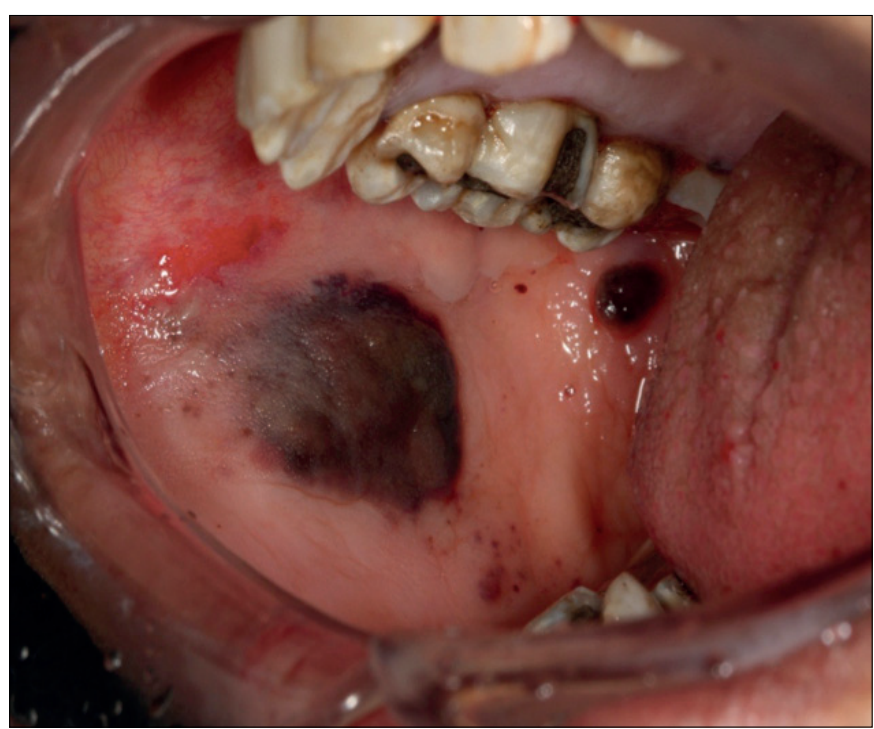

Fig. 6. Face interne de la joue droite.

Fig. 6. Internal side of the right cheek.

La patiente présentait un tableau hémorragique cutanéomuqueux diffus en l'absence de cause locale faisant évoquer un trouble systémique de l'hémostase. Les signes de gravité étaient : la nature spontanée des saignements, l'étendue de la surface corporelle atteinte, l'arrêt de la pilule contraceptive risquant de provoquer des ménorragies abondantes. Un avis spécialisé en hématologie a été demandé et la patiente a été transférée aux urgences médicales.

Une numération formule sanguine montrait une thrombopénie sévère à $4 \mathrm{~g} / \mathrm{L}(\mathrm{N}=150-400 \mathrm{~g} / \mathrm{L})$ ainsi qu'une anémie normocytaire régénérative : hémoglobine : $9 \mathrm{~g} / \mathrm{dL}(\mathrm{N}=13-18 \mathrm{~g} /$ $\mathrm{dL})$, VGM : $84(\mathrm{n}=80-100)$, taux de réticulocytes : $175 \mathrm{~g} / \mathrm{L}$

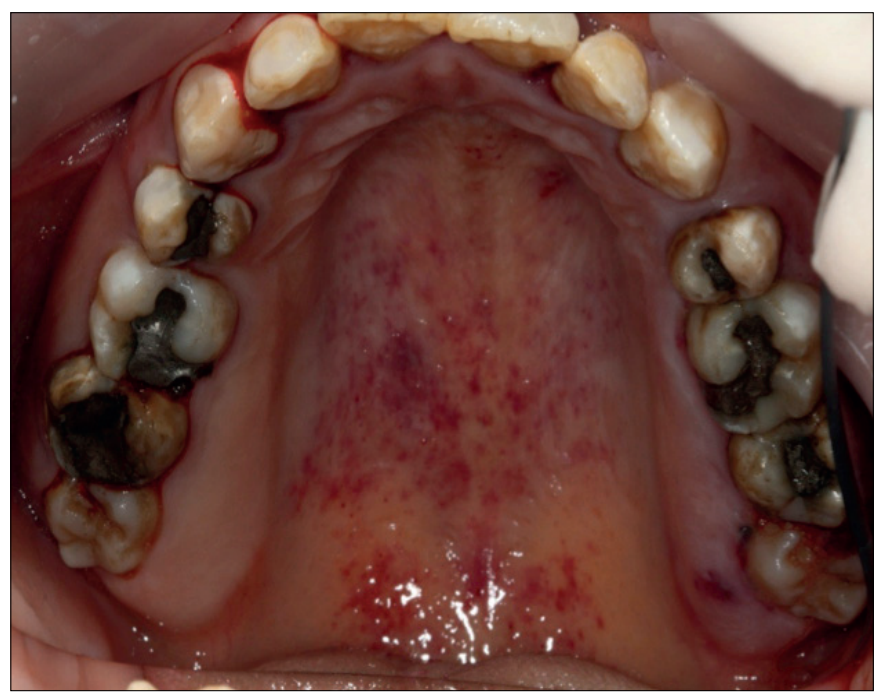

Fig. 7. Pétéchies palatines.

Fig. 7. Palatal petechia.

( $\mathrm{n}>150 \mathrm{~g} / \mathrm{L}$ ). Le taux de polynucléaires neutrophiles était normal: $\mathrm{PNN}=4500 / \mathrm{mm}^{3}\left(\mathrm{~N}=1600\right.$ à $\left.7000 / \mathrm{mm}^{3}\right)$.

Le bilan d'hémostase complet (TP, TCA, fibrinogène) ne présentait aucune anomalie. L'absence de troubles de la coagulation plasmatique et de la fibrinolyse nous a orientés vers un trouble de l'hémostase primaire pur ayant pour origine une thrombopénie sévère.

L'entretien médical et le taux normal de réticulocytes permettaient d'exclure une origine centrale de la thrombopénie. Une recherche systématique des différentes étiologies des thrombopénies périphériques a été effectuée : l'origine médicamenteuse avec la pilule contraceptive n'a pas été retenue (faible imputabilité intrinsèque et extrinsèque), les sérologies VIH, VHB, VHC, CMV ainsi qu'un MNI-test étaient négatifs. La patiente a déclaré ne pas consommer d'alcool. Le bilan d'hémostase réalisé permettait d'exclure une coagulopathie. Une échographie abdominale n'a pas retrouvé d'hépato-splénomégalie. La patiente n'a pas reçu de transfusion récente. La recherche d'une cause auto-immune par anticorps circulants (anticorps anti-cardiolipides, facteur rhumatoïde, anticorps anti-ß2-GP1) était négative. Le diagnostic de purpura thrombopénique immunologique a été retenu.

L'évaluation de la gravité du tableau a été faite sur le score hémorragique clinique supérieur à 8 (Tab. I [2,3]) et le taux de plaquettes de $4 \mathrm{~g} / \mathrm{L}$. Le traitement débuté en urgence fut le suivant : prednisone $1 \mathrm{mg} / \mathrm{kg}$ par jour per os, perfusion d'immunoglobulines $1 \mathrm{~g} / \mathrm{kg}$ à $\mathrm{J} 1$ (Tégéline ${ }^{\circledR}$ ). La pilule contraceptive a été reprise. Des bains de bouche d'acide tranexamique $\left(\operatorname{Exacy}^{\circledR}{ }^{\circledR}\right.$ ) ont été prescrits pour diminuer le saignement intrabuccal. Les ulcérations douloureuses ont été soulagées par un gel de xylocaïne visqueuse à $2 \%$. Des conseils d'hygiène buccodentaire dans ce contexte de gingivorragies ont été donnés. 
Tableau I. Score hémorragique repris par la HAS (d'après Khellaf, 2005 [3]). Table 1. Haemorragic score from HAS (from Khellaf 2005, [3]).

\begin{tabular}{|c|c|c|c|}
\hline \multicolumn{2}{|l|}{ Âge } & \multicolumn{2}{|l|}{ Saignement gastro-intestinal } \\
\hline Âge $>65$ ans & 2 & Saignement digestif sans anémie & 4 \\
\hline$\hat{\text { Age }}>75$ ans & 5 & $\begin{array}{l}\text { Saignement digestif avec anémie (perte de plus de } 2 \mathrm{~g} \\
\text { d'hémoglobine) et/ou choc }\end{array}$ & 15 \\
\hline \multicolumn{2}{|l|}{ Saignement cutané } & \multicolumn{2}{|l|}{ Saignement urinaire } \\
\hline Purpura pétéchial localisé (membres) & 1 & Hématurie macroscopique sans anémie & 4 \\
\hline Purpura ecchymotique & 2 & Hématurie macroscopique avec anémie aiguë & 10 \\
\hline Purpura pétéchial avec localisations multiples & 3 & \multicolumn{2}{|l|}{ Saignement du système nerveux central (SNC) } \\
\hline Purpura pétéchial généralisé & 3 & $\begin{array}{l}\text { Saignement du SNC ou saignement avec mise en jeu du } \\
\text { pronostic vital }\end{array}$ & 15 \\
\hline Purpura ecchymotique généralisé & 4 & & \\
\hline \multicolumn{2}{|l|}{ Saignements muqueux } & & \\
\hline Épistaxis unilatérale & 2 & & \\
\hline Épistaxis bilatérale & 3 & & \\
\hline Bulles hémorragiques & 5 & & \\
\hline
\end{tabular}

L'évolution a été marquée par des ménorragies abondantes le soir de la consultation ainsi que de nouvelles ecchymoses, une thrombopénie à $2 \mathrm{~g} / \mathrm{L}$ a été retrouvée le lendemain matin. Une seconde cure de Tégéline ${ }^{\circledR} 1 \mathrm{~g} / \mathrm{kg} / \mathrm{IV}$ a été administrée à $\mathrm{J} 2$. À J3, face à une numération plaquettaire à $8 \mathrm{G} / \mathrm{L}$ et une hémoglobine à $7,7 \mathrm{~g} / \mathrm{dL}$, une transfusion de deux culots globulaires a été réalisée dans le but de remonter l'hémoglobine de deux points (objectif en termes de numération : hémoglobine $=9,7 \mathrm{~g} / \mathrm{dL})$.

La patiente est sortie d'hospitalisation à $\mathrm{J7}$ avec une numération plaquettaire de $260 \mathrm{~g} / \mathrm{L}$ et une hémoglobine de $11,5 \mathrm{~g} /$ $\mathrm{dL}$. Le traitement de sortie fut le suivant : prednisone $20 \mathrm{mg}$, 3 comprimés le matin pendant 3 semaines ainsi qu'une contraception orale par Lutéran ${ }^{\circledR}, 1$ comprimé par jour.

La consultation de contrôle à $\mathrm{J} 15$ a montré une numération plaquettaire de $284 \mathrm{~g} / \mathrm{L}$ et une hémoglobinémie de $11,2 \mathrm{~g} / \mathrm{dL}$. Les lésions de la muqueuse buccale retrouvées à la phase initiale se sont réduites en nombre et en taille.

Une rechute à six semaines fut marquée par l'apparition d'un nouveau syndrome hémorragique cutanéo-muqueux. La numération plaquettaire était de $81 \mathrm{~g} / \mathrm{L}$ et le taux d'hémoglobine était de $12,1 \mathrm{~g} / \mathrm{dL}$.

La consultation de contrôle à deux mois a montré une muqueuse buccale parfaitement saine (Fig. 8) avec disparition totale de l'hématome, de la vésicule, des pétéchies et des gingivorragies.

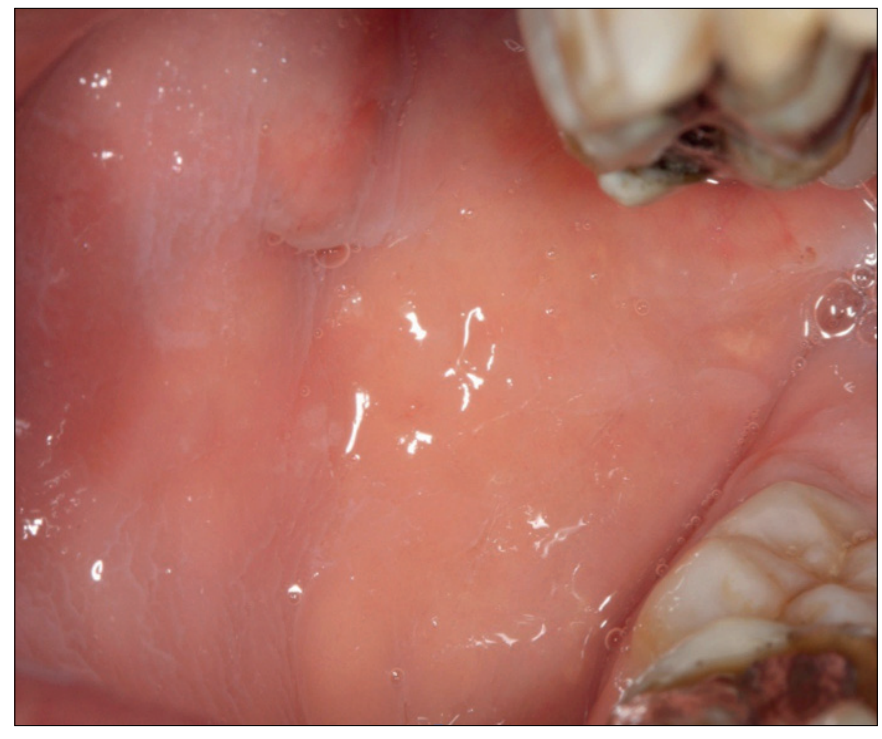

Fig. 8. Face interne de la joue droite 2 mois après.

Fig. 8. Internal side of the right cheek 2 months after.

\section{Discussion}

Le purpura thrombopénique immunologique (PTI) est caractérisé par la présence d'une thrombopénie (plaquettes 


\section{A S C L I N I Q U E}

Tableau II. Définitions des manifestations hémorragiques basées sur l'examen clinique (d'après Rodeghiero, 2013 [5]) Table II. Heamorragic manifestations from clinical exam (from Rodeghiero 2013 [5]).

\begin{tabular}{|l|l|l|}
\hline Site du saignement & Manifestation & Définition \\
\hline $\begin{array}{l}\text { Peau } \\
\text { (épiderme et derme) }\end{array}$ & Pétéchie & $\begin{array}{l}\text { Discoloration rouge (récente) ou violacée (de quelques jours) de la peau avec } \\
\text { un diamètre de 0,5 à 3 mm qui ne blanchit pas à la pression et qui n'est pas } \\
\text { palpable }\end{array}$ \\
\cline { 2 - 3 } & $\begin{array}{l}\text { Ecchymose (macule } \\
\text { purpurique, contusion) }\end{array}$ & $\begin{array}{l}\text { Tache plate, arrondie ou de forme irrégulière, rouge, bleue, violacée ou vert } \\
\text { jaunâtre, plus large qu'une pétéchie. La surélévation indique l'infiltration d'un } \\
\text { hématome sous-jacent dans les couches superficielles de la peau }\end{array}$ \\
\hline Peau (tissus sous-cutané) & Hématome & Accumulation localisée de sang souvent associée à une discoloration de la peau \\
\hline Muqueuses visibles & $\begin{array}{l}\text { Pétéchie, macule } \\
\text { purpurique et ecchymose }\end{array}$ & Idem que pour la peau \\
\cline { 2 - 3 } & $\begin{array}{l}\text { Bulles, vésicules } \\
\text { et cloques }\end{array}$ & $\begin{array}{l}\text { Lésion liquidienne avec soulèvement circonscrit contenant du sang } \\
\text { bulle > 5 mm, vésicule < } 5 \text { mm }\end{array}$ \\
\cline { 2 - 3 } & Épistaxis & Tout saignement du nez, antérieur ou postérieur, unilatéral ou bilatéral \\
\cline { 2 - 3 } & Gingivorragies & Tout saignement de la gencive marginale \\
\hline Muscles et tissus mous & Hématome & Toute collection de sang localisée, visible, palpable ou révélée par imagerie \\
\hline
\end{tabular}

$<150 \mathrm{~g} / \mathrm{L}$ ) causée par la présence d'auto-anticorps antiplaquettes qui entraînent leur destruction par les phagocytes mononucléés, principalement dans la rate et par un défaut de production médullaire d'origine immunologique [2].

L'incidence chez l'adulte est estimée à 1,6 cas pour 100000 sujets par an. Il existe une prédominance féminine modérée avec un sex ratio voisin de 1,3. Le PTI peut survenir à tout âge. L'étiologie du PTI est inconnue. Le PTI n'est pas une affection génétique, les formes familiales sont exceptionnelles [2]. On distingue trois formes de PTI [4] :

- le PTI nouvellement diagnostiqué depuis moins de trois mois ;

- le PTI persistant entre trois et douze mois après le diagnostic : une rémission spontanée peut survenir, mais il est impossible de se prononcer sur l'évolution à long terme ;

- le PTI chronique ayant plus de douze mois d'évolution : la probabilité de rémission ou de guérison spontanée est alors très faible (probablement inférieure à $5 \%$ ). Quatre-vingt pour cent des enfants atteints guérissent en quelques semaines. À l'inverse, le PTI évolue vers la chronicité chez $70 \%$ des adultes [2].

Le diagnostic de PTI est un diagnostic d'élimination. Il n'existe aucun test permettant d'affirmer le diagnostic.

L'examen clinique met en évidence des manifestations hémorragiques cutanées et muqueuses causées par la thrombopénie sévère : pétéchies, macules purpuriques, hématomes, ecchymoses, bulles et vésicules hémorragiques, épistaxis, gingivorragies, hémorragies sous-conjonctives. Dans le but de mieux caractériser ces signes cliniques, le groupe de travail international du PTI a mis à jour en 2013 les définitions des manifestations hémorragiques (Tab. II) [5]. Dans la situation clinique présentée, ce sont les gingivorragies qui ont amené la patiente à consulter les urgences odontologiques. Les macules purpuriques, les pétéchies et la bulle hémorragique nous ont orientés vers un trouble sévère de l'hémostase. Face à cette situation, il faut approfondir l'entretien médical pour évaluer la gravité du risque hémorragique à la recherche d'épistaxis, d'hématurie, de saignement digestif ou gynécologique, de céphalée inhabituelle $[6,7]$. L'examen des muqueuses buccales doit être complété par l'examen de la peau à la recherche de pétéchies cutanées ou d'hématome.

L'examen clinique doit être suivi par un ensemble d'examens complémentaires regroupant des examens biologiques, radiologiques, échographiques résumés dans le Tableau III.

La synthèse de ces examens permet de poser le diagnostic de PTI en éliminant :

- une thrombopénie centrale (causée par un syndrome myélodysplasique par exemple) ;

- une séquestration splénique en rapport avec une splénomégalie (étiologies diverses : hypertension portale, maladie de surcharge) ; dans cette situation, la thrombopénie est souvent modérée (rarement inférieure à $50 \mathrm{~g} / \mathrm{L}$ ) ;

- une consommation en rapport avec une coagulation intravasculaire ou une microangiopathie ;

- une cause périphérique immunologique secondaire à une infection virale (VIH, VHC) ou à une autre maladie auto-immune (en particulier un lupus) ou d'origine médicamenteuse (on parle alors de PTI associé) [2].

Chez l'adulte, les traitements de première ligne ont pour but de faire remonter rapidement le nombre des plaquettes.

Lorsque la NP est supérieure à $30 \mathrm{~g} / \mathrm{L}$, aucun traitement n'est nécessaire sauf :

- en cas de traitement antiagrégant ou anticoagulant concomitant ; 
Tableau III. Examens complémentaires à demander dans le contexte d'un PTI (d'après la HAS, 2009 [2]). Table III. Secondary exams that should be done in PTI context (from HAS 2009 [2]).

\begin{tabular}{|c|c|c|}
\hline Examens systématiques & Examens en fonction du contexte & Examens inutiles \\
\hline $\begin{array}{l}\text { - NFS sur tube citrate si doute sur fausse thrombopénie } \\
\text { à l'EDTA } \\
\text { - Frottis sanguin analyse par l'hématologiste biologiste } \\
\text { - Électrophorèse des protéines sériques ou dosage } \\
\text { pondéral des Ig } \\
\text { - Sérologies VIH } \\
\text { - Sérologies des hépatites B et C } \\
\text { - Bilan hépatique } \\
\text { - Anticorps antinucléaires } \\
\text { - TSH et anticorps antithyroïdes } \\
\text { - Créatinine TP, TCA, fibrinogène } \\
\text { - Groupe sanguin, agglutinines irrégulières dans les } \\
\text { formes sévères }\end{array}$ & $\begin{array}{l}\text { - Myélogramme +/- caryotype } \\
\text { - Recherche d'un anticoagulant circulant } \\
\text { - Anticorps anticardiolipides } \\
\text { - Chez l'adulte, recherche d'une infection par } \\
\text { Helicobacter pylori } \\
\text { - Échographie abdominale systématique pour } \\
\text { certains, en particulier si une splénectomie est } \\
\text { envisagée } \\
\text { - Immunophénotypage des lymphocytes } \\
\text { circulants } \\
\text { - Immunoélectrophorèse des protéines sériques } \\
\text { - Durée de vie isotopique des plaquettes } \\
\text { - Anticorps antiplaquettes par MAIPA }\end{array}$ & $\begin{array}{l}\text { - Temps de saignement } \\
\text { - Dosage du complément } \\
\text { - Dosage de TPO et recherche } \\
\text { de plaquettes réticulées }\end{array}$ \\
\hline
\end{tabular}

- chez le sujet âgé présentant une ou plusieurs comorbidités ;

- s'il existe une lésion susceptible de saigner ;

- en cas de signes hémorragiques.

Lorsque la NP est inférieure à $30 \mathrm{~g} / \mathrm{L}$, les traitements proposés sont :

- la corticothérapie par voie orale : prednisone à la dose de $1 \mathrm{mg} / \mathrm{kg} /$ jour pendant 2 à 3 semaines ;

- la transfusion de plaquettes est inutile voire dangereuse étant donné le mécanisme auto-immun à l'origine de la destruction plaquettaire ;

- la perfusion d'immunoglobulines intraveineuses : elle est indiquée uniquement en cas d'urgence hémorragique. La perfusion d'immunoglobulines humaines (Tégéline ${ }^{\circledR}$ ) en cas de PTI chez l'adulte est encadrée par l'établissement du score hémorragique reprit par la HAS en 2009 [2] regroupant les manifestations cliniques hémorragiques cutanéo-muqueuses (Tab. I). En cas de score inférieur à 8 , une prescription de corticoïdes seuls est proposée en première intention; si le score est supérieur à 8 , une prescription d'immunoglobulines adjuvante est recommandée [2]. Ce traitement a une efficacité toujours transitoire. Il doit donc être réservé aux situations où le syndrome hémorragique est important, comme dans le cas présenté. La dose habituelle est de 0,8 à $1 \mathrm{~g} / \mathrm{kg} / \mathrm{jour}$ chez l'adulte à $\mathrm{J} 1$;

- la transfusion plaquettaire : uniquement dans les cas avec mise en jeu du pronostic vital.

En cas de PTI chronique (plus de 12 mois) avec une NP $<30 \mathrm{~g} / \mathrm{L}$, le traitement de référence est la splénectomie [2].

La surveillance est basée sur deux paramètres essentiels : la numération plaquettaire et le score hémorragique. Les signes cliniques hémorragiques sont d'excellents indicateurs de la sévérité de la thrombopénie dans le cadre du PTI permettant d'objectiver la susceptibilité du patient face à cette thrombopénie.
Dans le cas présenté, l'association de bulles hémorragiques intrabuccales ainsi que la présence d'un hématome jugal étaient à considérer comme des signes de gravité.

Au décours de l'épisode aigu, le patient doit être informé de la nécessité de réaliser une NFS (avec une numération plaquettaire) en cas de saignement, ou avant chaque manœuvre invasive (intervention chirurgicale, soins dentaires avec extraction, acte endoscopique, injection intramusculaire) [8].

\section{Conclusion}

Lorsque l'examen clinique des muqueuses buccales révèle la présence de lésions hémorragiques (pétéchies, purpura, bulles hémorragiques), il faut systématiquement évaluer la gravité du trouble de l'hémostase en approfondissant l'anamnèse médicale. Il faut dépister des saignements provoqués ou spontanés (épistaxis, hématurie) et réaliser un examen clinique plus global à la recherche de lésions hémorragiques cutanées. La présence de lésions hémorragiques buccales est un signe de gravité, elle correspond à une situation d'urgence médicale. Le PTI entraîne une thrombopénie sévère brutale à l'origine de l'apparition de ces lésions qui sont en général les premiers signes de l'affection. Le traitement de première intention est la corticothérapie par voie orale pouvant être complétée par l'injection d'immunoglobulines dans les formes sévères. Chez l'adulte, une fois la phase aiguë passée, le PTI évolue vers la chronicité dans $70 \%$ des cas. Une surveillance est indispensable et l'apparition de lésions hémorragiques buccales est l'un des premiers signes de récidive.

\section{Conflits d'intérêt : aucun}




\section{A S C L I N I Q U E}

\section{Références}

1. Provan D, Stasi R, Newland AC, Blanchette VS, BoltonMaggs $P$, Bussel JB, et al. International consensus report on the investigation and management of primary immune thrombocytopenia. Blood 2010;115:168-186.

2. Haute Autorité de Santé. Purpura thrombopénique immunologique de l'enfant et de l'adulte. Protocole national de diagnostic et de soins. Octobre 2009.

3. Khellaf $M$, Michel M, Schaeffer A, Bierling P, Godeau B. Assessment of a therapeutic strategy for adults with severe autoimmune thrombocytopenic purpura based on a bleeding score rather than platelet count. Haematologica 2005;90:829-832.
4. Rodeghiero F, Stasi R, Gernsheimer T, Michel M, Provan D, Arnold DM, et al. Standardization of terminology, definitions and outcome criteria in immune thrombocytopenic purpura of adults and children: report from an international working group. Blood 2009;113:2386-2393.

5. Rodeghiero $F$, Michel $M$, Gernsheimer $T$, Ruggeri $M$, Blanchette V, Bussel JB, et al. Standardization of bleeding assessment in immune thrombocytopenia: report from the International Working Group. Blood 2013;121:2596-2606.

6. Kayal L, Jayachandran S, Singh K. Idiopathic thrombocytopenic purpura. Contemp Clin Den 2014;5:410-414.

7. Themistocleous E, Ariyaratnam S, Duxbury AJ. Acute idiopathic thrombocytopenic purpura: a case report. Dent Update 2004;31:92-96.

8. 8. Guzeldemir E. The role of oral hygiene in a patient with idiopathic thrombocytopenic purpura. Int $\mathrm{J}$ Dent Hyg $2009 ; 7: 289-293$. 